\title{
Blast to Leukocyte Ratio
}

National Cancer Institute

\section{Source}

National Cancer Institute. Blast to Leukocyte Ratio. NCI Thesaurus. Code C64487.

The determination of the ratio of blasts compared to leukocytes in a blood sample. The result may be expressed as either a ratio or a percentage. 